\title{
Orthodontic retention to have and to hold
}

\section{Abstracted from \\ Littlewood SJ, Millett DT, Doubleday B, Bearn DR, Worthington HV.}

Retention procedures for stabilising tooth position after treatment with orthodontic braces. Cochrane Database Syst Rev 2016; 1: Art. No.: CD002283. DOI: 10.1002/14651858.CD002283.pub4.

Address for correspondence: Luisa Fernandez Mauleffinch, Managing Editor, Cochrane Oral Health Group, School of Dentistry, The University of Manchester, JR Moore Building, Oxford Road, Manchester, M13 9PL, UK. E-mail: luisa.fernandez@manchester.ac.uk

\section{Question: What is the best method for maintaining the correct position of teeth after orthodontic treatment?}

Data sources Cochrane Oral Health Group's Trials Register, Cochrane Central Register of Controlled Trials (CENTRAL), Medline, Embase, US National Institutes of Health Trials Registry and The World Health Organization (WHO) Clinical Trials Registry Platform, abstracts from the British Orthodontic Conference, the European Orthodontic Conference and the International Association for Dental Research (IADR) from 2011 to 2015 and the bibliographies of identified studies. Study selection Randomised controlled trials (RCTs) involving children and adults who had had retainers fitted or adjunctive procedures undertaken to prevent relapse following orthodontic treatment with braces were considered.

Data extraction and synthesis Two reviewers independently selected studies, abstracted data and assessed study quality. For continuous data mean differences $(\mathrm{MD})$ with $95 \%$ confidence intervals $(\mathrm{Cl})$ were calculated with ratios (RR) and $95 \% \mathrm{Cl}$ for dichotomous outcomes. Results Fifteen studies involving a total of 1722 patients were included. Seven studies were considered to be at high risk of bias, four at low risk and four at unclear risk. For removable retainers versus fixed retainers (three studies) there was low quality evidence that thermoplastic removable retainers provided slightly poorer stability in the lower arch than multistrand fixed retainers: MD (Little's Irregularity Index, $0 \mathrm{~mm}$ is stable) $0.6 \mathrm{~mm}(95 \% \mathrm{Cl} 0.17$ to 1.03$)$ and of less gingival bleeding with removable retainers: RR 0.53 (95\% Cl; 0.31 to 0.88). Patients found fixed retainers more acceptable to wear, with a mean difference on a visual analogue scale (VAS; 0 to 100; 100 being very satisfied) of -12.84 ( $95 \% \mathrm{Cl}-7.09$ to -18.60$)$.

For different types of fixed retainers (four studies) data from three studies (228 patients) comparing polyethylene ribbon bonded retainer versus multistrand retainer were pooled showing no evidence of a difference in failure rates. $\mathrm{RR}=1.10(95 \% \mathrm{Cl} ; 0.77$ to 1.57$)$.

Pooled data from two trials (174 patients) comparing the same types of upper fixed retainers, showed a similar finding: $\mathrm{RR}=1.25(95 \% \mathrm{Cl}$; 0.87 to 1.78$)$.

This paper is based on a Cochrane Review published in the Cochrane Library 2016, issue 4 (see www.thecochranelibrary.com for information). Cochrane Reviews are regularly updated as new evidence emerges and in response to feedback, and the Cochrane Library should be consulted for the most recent version of the review.
For different types of removable retainers (eight studies) one study at low risk of bias comparing upper and lower part-time thermoplastic versus full-time thermoplastic retainers showed no evidence of a difference in relapse (graded moderate quality evidence). Another study, comparing part-time and full-time wear of lower Hawley retainers, found no evidence of any difference in relapse (low quality evidence). Two studies at high risk of bias suggested that stability was better in the lower arch for thermoplastic retainers versus Hawley, and for thermoplastic full-time versus Begg (fulltime) (both low quality evidence). In one study, participants wearing Hawley retainers reported more embarrassment more often than participants wearing thermoplastic retainers: RR 2.42 ( $95 \% \mathrm{Cl} 1.30$ to 4.49 ; one trial, 348 participants, high risk of bias, low quality evidence). They also found Hawley retainers harder to wear. There was conflicting evidence about survival rates of Hawley and thermoplastic retainers.

For combination of upper thermoplastic and lower bonded versus upper thermoplastic with lower adjunctive procedures versus positioner (one study) there was no evidence of a difference in relapse between the combination of an upper thermoplastic and lower canine to canine bonded retainer and the combination of an upper thermoplastic retainer and lower interproximal stripping, without a lower retainer. Both these approaches are better than using a positioner as a retainer.

Conclusions We did not find any evidence that wearing thermoplastic retainers fulltime provides greater stability than wearing them part-time, but this was assessed in only a small number of participants. Overall, there is insufficient high quality evidence to make recommendations on retention procedures for stabilising tooth position after treatment with orthodontic braces. Further high quality RCTs are needed.

\section{Commentary}

A new systematic review on retention arouses both curiosity and pique for any practitioner of the art and science of orthodontics. Retention continues to be the quintessential enigma with each practitioner claiming a fair share of success and failure.

It is the intention of every orthodontist to enter into something similar to a marriage vow with a treated malocclusion 'to have and to hold forever'. But retention continues to be a grey area with empirical directions and claims. This review does address a direct question on which is the best method of retaining teeth after orthodontic treatment. The review is based on the inclusion of 15 studies with 1722 patients selected from a pool of scientific data 
generated from a very cohesive and comprehensive search strategy. All the possible databases have been covered, with two reviewers independently searching, studying and selecting the studies. A meta-analysis has been done where the studies have shown similar methodology and analysis of similar outcomes. The Little's irregularity index has been prioritised to measure relapse, but the detailed analysis includes stability parameters defined by upper and lower inter canine and inter molar arch widths, arch length, patient satisfaction, loss and frequency of wear. It's a huge mandate, and knowing the disparity in published literature it would be difficult to concur on a significant outcome.

Various strategies have been written about and described in literature but there are some discerning trends that come to the fore. The increased usage of vacuum formed retainers addressing both patient ease as well as the aesthetic implications is apparent.

Adjunctive procedures have been attempted before, permanent retention using the bonded lingual retainers finds an increasing usage, the traditional Hawley retainer still finds its place in the spectrum of appliances.

The review is complex because of the variables and the effort of the authors is to be appreciated to traverse this difficult terrain of variable interventions and outcomes. The first and most obvious comparison would be between removable and fixed retainers. The lower thermoplastic retainers provide comparatively less stability than the lower bonded multi strand retainers. Bond failures compromise the bonded retainers and the possible effect on gingival bleeding. The patient acceptability of the aesthetic VFR is obviously more. So what clinical recommendations would be forthcoming form this evidence? Would an upper thermoplastic retainer work better with a lower bonded retainer? The studies included in this review do not provide any leading answers. If one is to compare the use of thermoplastic retainers against the Hawley or the Begg wraparound retainer, then the thermoplastic retainer seems to score marginally better. Could this be attributed to the easier acceptability of the aesthetic VFR? After all, compliance is a major issue in all removable appliances and would have a clear impact.
The best material type for bonded retainers also has no clarity; essentially the polyethylene ribbon and the multi stranded wire retainers show an equitable number of debonds. One of the issues that any discerning orthodontist would like to take away from a large and complex review is the issue of part time wear of retainers versus the full time wear. Here there is some evidence, however limited, that part time wear is as effective as full time wear of the retainers.

The complex review leaves one with a feeling of grasping for straws in the wind. The conclusion is as expected, lamenting the lack of good quality evidence to make an evidence based recommendation of the best method of retention. But the authors do find it cogent to summarise that full time wear of thermoplastic retainers does not provide any additional benefit over part time wear. The results however do show some very useful trends even if the evidence is lacking. The thermoplastic retainers come with aesthetic benefits which would improve compliance and wearing of the retainers for whatever period the orthodontist recommends. The issue would be the interpostioning in the buccal regions where the teeth would not permit a setting of the buccal segments. Would this be desirable in correction of buccal segments from class 2 to class 1 where a good cusp fossa relation would add to stability? The Hawley or wraparound retainer would facilitate such a settling of buccal segments. The issue of lower anterior crowding would need a clinical direction where a correction of rotations with a resolution of a Bolton discrepancy may be retained with a vacuum formed upper retainer only. The review is complex, carefully crafted, backed on a thorough search and qualitative assessment strategy. The diversity of evidence is only to be expected. But answers to the enigma of retention will be elusive. Suffice it to say that evidence based retention protocols are not in sight. One cannot but help agree with the authors about the need for qualitative research.

Anmol S Kalha

ITS Dental College, Hospital and Research Center, Greater Noida, NCR, India.

Evidence-Based Dentistry (2016) 17, 105-106. doi:10.1038/sj.ebd.6401200 